\title{
A Cross-Cutting Approach to Surveillance and Laboratory Capacity as a Platform to Improve Health Security in Uganda
}

Mohammed Lamorde, Arthur Mpimbaza, Richard Walwema, Moses Kamya, James Kapisi, Henry Kajumbula, Asadu Sserwanga, Jane Frances Namuganga, Abel Kusemererwa, Hannington Tasimwa, Issa Makumbi, John Kayiwa, Julius Lutwama, Prosper Behumbiize, Abner Tagoola, Jane Frances Nanteza, Gilbert Aniku, Meklit Workneh, Yukari Manabe, Jeff N. Borchert, Vance Brown, Grace D. Appiah, Eric D. Mintz, Jaco Homsy, George S. Odongo, Raymond L. Ransom, Molly M. Freeman, Robyn A. Stoddard, Renee Galloway, Matthew Mikoleit, Cecilia Kato, Ronald Rosenberg, Eric C. Mossel, Paul S. Mead, and Kiersten J. Kugeler

\footnotetext{
Mohammed Lamorde, PhD, FRCP, is Head of the Department of Prevention, Care and Treatment, Infectious Diseases Institute, Kampala, Uganda. Arthur Mpimbaza, MBChB, MMed, MSc, is Project Coordinator, Infectious Diseases Research Collaboration, Kampala, and Lecturer, Child Health and Development Centre, College of Health Sciences, Makerere University, Kampala. They are co-senior authors. Richard Walwema, MBA, is with the Infectious Diseases Institute, Kampala. Moses Kamya, MBChB, MMed, $\mathrm{MPH}, \mathrm{PhD}$, is Professor and Chair; and Henry Kajumbula, MBChB, MMed, and Hannington Tasimwa are in the Department of Medical Microbiology; all at Makerere University School of Medicine, Kampala. Dr. Kamya is also with the Infectious Diseases Research Collaboration, Kampala. James Kapisi, MBChB, MMed, MSc Epid; Asadu Sserwanga, MBChB, MPH; Jane Frances Namuganga, MPH; and Abel Kusemererwa are all with the Infectious Diseases Research Collaboration, Kampala. Issa Makumbi, $\mathrm{MBChB}$, is with the Uganda Ministry of Health Public Health Emergency Operations Centre, Kampala. John Kayiwa and Julius Lutwama, PhD, are with the Uganda Virus Research Institute, Entebbe, Wakiso, Uganda. Prosper Behumbiize is with the Health Information Systems Program, Kampala. Abner Tagoola, MBChB, MMed, MSc, is with the Jinja Regional Referral Hospital Republic of Uganda Ministry of Health, Jinja, Uganda. Jane Frances Nanteza, MBChB, MMed, is with the Mubende Regional Referral Hospital, Republic of Uganda Ministry of Health, Mubende, Uganda. Gilbert Aniku, MBChB, MMed, is with Arua Regional Referral Hospital, Republic of Uganda Ministry of Health, Arua, Uganda. Meklit Workneh, MD, MPH, is a Medical Microbiology Fellow, and Yukari Manabe, MD, is Associate Director of Global Health Research and Innovation, both at the Johns Hopkins School of Medicine, Baltimore, Maryland. Jeff N. Borchert, MS, is a Public Health Analyst; Ronald Rosenberg, ScD, is Associate Director for Science; Eric C. Mossel, PhD, is a Microbiologist; and Paul S. Mead, MD, MPH, is a Medical Officer; all at the Centers for Disease Control and Prevention, Division of Vector-Borne Diseases, Fort Collins, Colorado. Vance Brown, MA, is Deputy Program Director, and Jaco Homsy, MD, MPH, is Program Director, Centers for Disease Control and Prevention, Division of Global Health Protection, Kampala, Uganda. Grace D. Appiah, MD, MS, is a Medical Epidemiologist; Eric D. Mintz, MD, MPH, is a Medical Epidemiologist; and Molly M. Freeman, PhD, is a Microbiologist; all in the Division of Foodborne, Waterborne, and Environmental Diseases, Centers for Disease Control and Prevention, Atlanta, Georgia. George S. Odongo, MPH, is a Public Health Informatics Fellow; Raymond L. Ransom is Associate Director for Informatics; and Matthew Mikoleit, MS, is a Microbiologist; all in the Division of Global Health Protection, Centers for Disease Control and Prevention, Atlanta, Georgia. Robyn A. Stoddard, DVM, PhD, and Renee Galloway, MLS, MPH, are Microbiologists, both in the Division of High-Consequence Pathogens and Pathology, Centers for Disease Control and Prevention, Atlanta, Georgia. Cecilia Kato, PhD, is a Biologist, Division of Vector-Borne Diseases, Centers for Disease Control and Prevention, Atlanta, Georgia. Kiersten Kugeler, PhD, MPH, is an Epidemiologist, Division of Vector-Borne Diseases, Centers for Disease Control and Prevention, Fort Collins, CO.
} 
Global health security depends on effective surveillance for infectious diseases. In Uganda, resources are inadequate to support collection and reporting of data necessary for an effective and responsive surveillance system. We used a cross-cutting approach to improve surveillance and laboratory capacity in Uganda by leveraging an existing pediatric inpatient malaria sentinel surveillance system to collect data on expanded causes of illness, facilitate development of real-time surveillance, and provide data on antimicrobial resistance. Capacity for blood culture collection was established, along with options for serologic testing for select zoonotic conditions, including arboviral infection, brucellosis, and leptospirosis. Detailed demographic, clinical, and laboratory data for all admissions were captured through a web-based system accessible at participating hospitals, laboratories, and the Uganda Public Health Emergency Operations Center. Between July 2016 and December 2017, the expanded system was activated in pediatric wards of 6 regional government hospitals. During that time, patient data were collected from 30,500 pediatric admissions, half of whom were febrile but lacked evidence of malaria. More than 5,000 blood cultures were performed; $4 \%$ yielded bacterial pathogens, and another $4 \%$ yielded likely contaminants. Several WHO antimicrobial resistance priority pathogens were identified, some with multidrug-resistant phenotypes, including Acinetobacter spp., Citrobacter spp., Escherichia coli, Staphylococcus aureus, and typhoidal and nontyphoidal Salmonella spp. Leptospirosis and arboviral infections (alphaviruses and flaviviruses) were documented. The lessons learned and early results from the development of this multisectoral surveillance system provide the knowledge, infrastructure, and workforce capacity to serve as a foundation to enhance the capacity to detect, report, and rapidly respond to wide-ranging public health concerns in Uganda.

Keywords: Global health security, Uganda, Surveillance

$\mathrm{F}$ EVER MAY be THE INITIAl OR SOle SYMPtom of many infectious diseases, including some with outbreak potential. ${ }^{1,2}$ Although clinical practice in many malaria-endemic areas has been to presumptively treat febrile patients for malaria, improved access to malaria diagnostics in recent years has revealed that a substantial proportion of acutely ill, febrile patients in sub-Saharan Africa do not have malaria. ${ }^{3-5}$ Yet, many countries lack resources and capacity for accurate diagnosis of most infectious conditions. Rapid response to diverse and emerging public health threats is severely challenged by lack of appropriate laboratory capacity and timely surveillance networks. ${ }^{6}$ These gaps foster antimicrobial and antimalarial resistance, limit evidence-based care and policy to improve population health, and hinder the ability to detect outbreaks early.

Uganda, an inland East African country with a rapidly growing population estimated at 43 million people in 2017, has made progress in recent decades to improve life expectancy, reduce poverty and food insecurity, and expand access to immunizations and clean water. ${ }^{7}$ Yet, as with many African countries, Uganda faces diverse health challenges in a weak health infrastructure that limits the rapid detection and confirmation of infections with epidemic potential. In the past 2 decades, Uganda has experienced outbreaks of emerging and reemerging infectious diseases including Ebola, Marburg, Crimean-Congo hemorrhagic fever, Rift Valley fever, yellow fever, hepatitis E, cholera, typhoid fever, plague, and anthrax. ${ }^{8-16}$

Effective laboratory capacity and disease surveillance are critical to global health security and the basis for the 2005 International Health Regulations (IHR) signed by all
World Health Organization (WHO) member states. IHR compliance has proven challenging for many low-income countries, including Uganda. ${ }^{17,18}$ The Global Health Security Agenda (GHSA), a multisectoral and multilateral partnership intended to support countries toward IHR compliance, launched officially in 2014 (www.ghsagenda. org). The government of Uganda and the US Centers for Disease Control and Prevention (CDC) jointly implemented a demonstration project a year earlier, in 2013. The pilot project improved specimen referral networks and information systems associated with outbreak response and created an emergency operations center; these activities set the stage for multiple GHSA activities in the country. ${ }^{19}$

Through GHSA-initiated partnerships, we introduced a cross-cutting surveillance approach to advance ability to detect unusual health events in Uganda. As conceived, this effort would provide comprehensive patient data and facilitate electronic systems infrastructure that could ultimately improve early detection of novel infections or outbreaks, define conditions causing human illness to inform appropriate and targeted laboratory capacity building efforts, and generate data for an antimicrobial resistance surveillance and intervention program in its infancy.

\section{Methods}

We modified an existing inpatient pediatric malaria surveillance system to provide patient data from all children 14 years old and younger who were admitted to 6 government hospitals, in concert with improved diagnostic ability to 
define causes of nonmalarial illness affecting the population. Five of the 6 designated hospitals have participated in ongoing pediatric malaria surveillance since 2010; 1 site in northwestern Uganda was added to improve geographic representation for this expanded system. Included hospitals reflected both district and regional hospitals, 2 tiers in the organizational structure of hospitals in Uganda. The Uganda Ministry of Health (designated sentinel hospital surveillance sites, Uganda Virus Research Institute, or UVRI, and the Uganda National Health Laboratory Services) partnered with a consortium composed of the Infectious Diseases Institute (IDI), Makerere University Department of Medical Microbiology, the Infectious Diseases Research Collaboration (IDRC), and the Health Information Systems Program (HISP-Uganda) for the capacity-building efforts. Standard operating procedures regarding data capture, phlebotomy, and specimen labeling, storage, and transport were developed or revised as necessary. Before implementation, plans were finalized by all partners, approved as public health surveillance/nonresearch by CDC (NCEZID \#031416), and approved by the Director General of Health Services, Ministry of Health, Uganda.

\section{Baseline Capacity Assessment, Training, and Site Activation}

Surveillance for clinical and demographic data was ongoing at 5 of the 6 sites via the inpatient malaria surveillance system. Thus, routine and standard data collection for purposes of surveillance was a new activity for only 1 site. Site activation reflected collection of expanded information for surveillance, improved electronic surveillance infrastructure, and laboratory diagnostic capacity. Activation of sites occurred between July 2016 and October 2017. The 6 sentinel hospital sites and their associated system activation dates were: Jinja Regional Referral Hospital (Jinja RRH) (July 2016), Arua RRH (July 2016), Mubende RRH (August 2016), Kabale RRH (April 2017), Apac District Hospital (July 2017), and Tororo District Hospital (October 2017) (Figure 1).

Before activation of this system at each selected government hospital, a baseline rapid assessment was conducted to determine: (1) existing on-site laboratory capacity for microbiology; (2) potential for expansion of on-site microbiologic capacity in terms of workforce, training needs, equipment needs, and available space; (3) biosafety and biosecurity practices and gaps; (4) infection prevention and control practices and gaps; and (5) existing organizational and management structures and their associated ability to support modified practices.

Briefly, assessment was conducted using the Potter and Brough Capacity Pyramid approach, which assesses capacities at various structural levels, all within the local context, including tools, skills, staff and infrastructure, structures, systems, and roles. ${ }^{20}$ Standards and tools used or adapted included those of the WHO SLIPTA program (Stepwise Laboratory Improvement Process Towards Accreditation), the WHO laboratory assessment tool, and CDC infection control assessment tools. ${ }^{21-23}$ These assessments revealed extensive gaps including inactive hospital-based infection prevention and control programs, lack of supplies and infrastructure to promote basic hand hygiene, lack of microbiology services, poor access control to laboratory rooms, inadequate sample transportation for microbiologic samples to a central reference laboratory, lack of defined systems for pharmaceutical tracking, weak information technology and data management, and inadequate interface between clinicians and hospital laboratories. Prior to activation, ongoing routine diagnostic testing was unavailable for infectious conditions other than malaria and HIV.

The process of surveillance site activation included inception meetings with varied hospital and department administrators and staff; provision of equipment and supplies for phlebotomy, infection control, clinical specimen processing and storage, and internet connectivity according to needs identified on assessment. At each surveillance site, multifaceted training was aimed at pediatricians, nurses, and laboratory technicians, but it included additional cadres of Ugandan government healthcare workers staffed in each hospital. This training was delivered through interactive lecture and hands-on practice. Content included aseptic technique, appropriate sample volumes for laboratory tests, and the importance of good record keeping for surveillance and improved clinical care. Training also included the importance of investigating nonmalarial causes of illness and incorporation of bacterial blood culture into routine clinical care. Initial emphasis focused on improving diagnosis of nonmalarial illnesses by reserving blood culture for febrile children without evidence of malaria by microscopy or rapid diagnostic test (RDT). However, clinicians were also encouraged to use blood culture as a diagnostic tool in any circumstance deemed appropriate.

Continued regular site support visits occurred following activation to provide ongoing training in phlebotomy, troubleshoot staff concerns, identify areas for improvement, and provide summary data and feedback. Concerns and issues that arose were addressed and incorporated into ongoing training efforts at all surveillance sites. When clustering of contaminated cultures in space and time occurred, investigation and intervention followed. Areas dedicated to phlebotomy were created on all wards to improve the likelihood of aseptic draws in otherwise difficult hygienic conditions.

\section{Laboratory System Strengthening}

Blood cultures were made available at the time of surveillance site activation. With a focus on improving surveillance and diagnostic capacity for severe illness in the pediatric population, most blood culture bottles purchased 


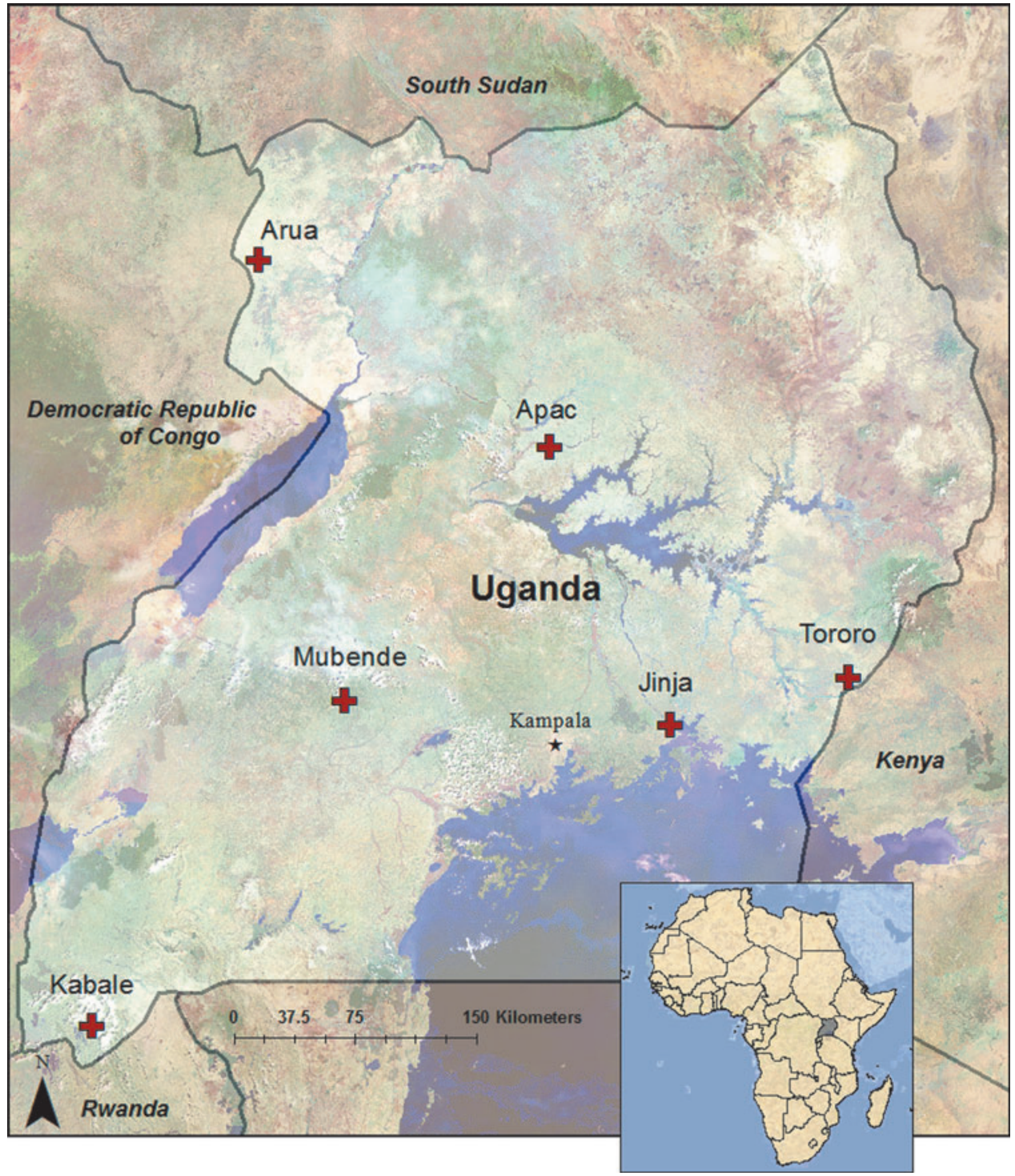

Figure 1. Geographic location of 6 sentinel site hospitals engaged in case-based surveillance and laboratory capacity-building efforts in Uganda

and provisioned to hospitals were aerobic pediatric bottles for blood volumes up to $3 \mathrm{~mL}$ according to patient age and weight. Before installation of BD BACTEC ${ }^{\mathrm{TM}}$ (Becton Dickinson, Franklin Lakes, NJ, USA) machines in sentinel hospital laboratories, all inoculated blood culture bottles were transported daily at ambient temperature to the Department of Medical Microbiology (DMM) at Makerere University in the capital city of Kampala for processing. Upon successful installation of BACTEC machines in sentinel site hospitals and associated training, those hospital laboratories incubated all blood culture bottles locally for up to 7 days. Negative results were provided directly back to hospital clinicians. Bottles read as positive by the machines were usually subjected to Gram stain at the hospital laboratory in order to provide timely and clinically relevant information before transportation to DMM for subculturing, identification, and antimicrobial susceptibility screening. Identification at DMM was performed using conventional, phenotypic identification algorithms down to species and subspecies or serotype when appropriate reagents were available. Classification of isolated organisms as pathogens or likely contaminants was made presumptively by DMM based on general knowledge regarding pathogenicity of various organisms and presented accordingly here. Most antimicrobial susceptibility testing was performed using the Kirby-Bauer disk diffusion method according to CLSI standards and interpreted according to 2014 breakpoints.

We instituted serologic tests for patients with a history of febrile illness of at least 4 days' duration, a restriction designed to assure improved sensitivity for detection of $\operatorname{IgM}$ antibodies for certain conditions. Tests were instituted at each site as they became available in Uganda and following adequate staff training, independent of site activation. A 
lateral flow assay to detect $\operatorname{IgM}$ antibody to a variety of Leptospira spp. was provided to all sentinel hospitals (Test$\mathrm{It}^{\mathrm{TM}}$, Life Assay, Cape Town, South Africa). Remaining sera were transported to the Uganda Virus Research Institute and screened for IgM reactivity to multiple mosquito-borne arboviruses, specifically West Nile, yellow fever, dengue serotypes 1-4, Zika, and chikungunya viruses. IgM positive samples were considered presumptive positive flavivirus or alphavirus infections. When sample volume allowed, IgM screening was followed with confirmatory plaque reduction neutralization tests (PRNT). This allowed for confirmation of specific infecting virus and differentiation of chikungunya and o'nyong nyong virus infections among those that screened positive on chikungunya IgM screening. Additionally, sera were tested for antibodies to Brucella abortus, B. suis, and B. melitensis by the microagglutination test, ${ }^{24}$ with minor modifications including use of U-bottom plates, incubation at $28^{\circ} \mathrm{C}$, and discontinued use of safranin, using antigen produced by the US Department of Agriculture National Veterinary Services Laboratory (Ames, Iowa, USA). Brucella antibody titers were interpreted as follows: $>160$ considered positive, 20-80 considered borderline, and $<20$ was considered negative. Remaining sera were stored for ultimate shipment to US CDC. Testing at CDC provided quality assurance of tests newly implemented in Uganda and allowed for investigation into other vector-borne and zoonotic conditions causing human illness in Uganda for which local laboratory diagnostic capacity had not yet been developed. Accordingly, sera were screened for human antibody reactivity to Rickettsia africae because of this antigen's apparent broad specificity for spotted-fever group rickettsiae (SFGR) in this geographic region (unreported data). Due to limits of available assays, screening was conducted using an immunofluorescence assay (IFA) that primarily detects IgG antibodies. Thus, this rickettsial screening was most likely to define past exposure rather than cause of current infection, nevertheless informing potential need for development of appropriate laboratory capacity in Uganda. A titer of 1:64 was considered the cut-off for significant antibody response.

\section{Surveillance and Laboratory Data Capture and Management}

In each sentinel hospital pediatric ward, a standardized medical record form previously developed for malaria surveillance $^{25}$ was adapted to incorporate additional variables relevant to expanded diagnostic capacity with stakeholder and clinician input. This form serves as a medical chart for all admissions, allowing providers to record symptoms, signs, treatments, and outcome at discharge.

A full-time surveillance data officer was stationed at each hospital. The primary functions of this person were to engage with clinicians and laboratory staff to ensure that samples were collected, labeled, and transported properly and that clinical and laboratory records were complete; to enter demographic, clinical, and laboratory data into the electronic database; and to facilitate ongoing communication among the surveillance team and hospital staff and administrators. Initially, data were entered into a Microsoft Access ${ }^{\circledR}$ database, but following improvements in connectivity, data entry was subsequently transitioned to a webbased data management system on the District Health Information System (DHIS-2) platform (www.dhis2.org). DHIS-2 is a flexible, open-sourced information system used by Uganda's Ministry of Health for aggregate public health data management and by the Public Health Emergency Operations Center for recording and tracking casebased data related to outbreak response and specimen collection, transportation, and testing. The system was tailored to allow case-based routine surveillance data transmission, with multiple data entry points on the same individual record to enable linking of patient data with central laboratory results, including antimicrobial resistance patterns. The system permits site-based staff, central laboratory staff, and ministry of health personnel to view data on individual patients and associated laboratory results in real-time, thereby improving data quality and use for case management, specimen tracking and testing, and disease surveillance.

\section{REsults}

\section{Clinical and Demographic Data}

Between July 2016 and December 2017, patient data were collected for 30,500 admissions in 6 hospitals (Table 1). Median patient age was 2 years (range: 1 day to 14 years); $56 \%$ were male. Of admitted patients, $74 \%(n=22,684)$ had on-schedule or complete childhood immunizations as assessed by providers on site. Most admissions $(n=26,573$; $87 \%)$ were associated with documented fever $\left(\geq 37.5^{\circ} \mathrm{C}\right)$ or history of fever. In accordance with national and international guidelines, most admissions for acute febrile illness $(87 \%)$ were tested for malaria by blood smear, rapid diagnostic test, or both $(n=26,526)$. Overall prevalence of malaria among these hospitalized patients was $53 \%$, ranging between $4 \%$ and $79 \%$ across hospitals (Table 1). Cough was reported for $62 \%(n=18,839)$ of admitted patients (range: 58\%-68\% across hospitals), and diarrhea was reported for $27 \%(n=8,151)$ patients (range: $20 \%-47 \%$ across hospitals). Gentamicin was the most commonly prescribed antibiotic, documented as treatment in more than 10,000 admissions; ceftriaxone was used in more than 8,500 admissions and ampicillin in more than 5,000 admissions.

Among 30,500 total admissions electronically captured between July 2016 and December 2017 at all 6 hospitals, 21,199 admissions (70\%) occurred after blood culture capacity had been established in each respective hospital and additional associated variables were being collected. Accordingly, this reflected all admissions from the first sites activated in July and August 2016 and only a fraction from 


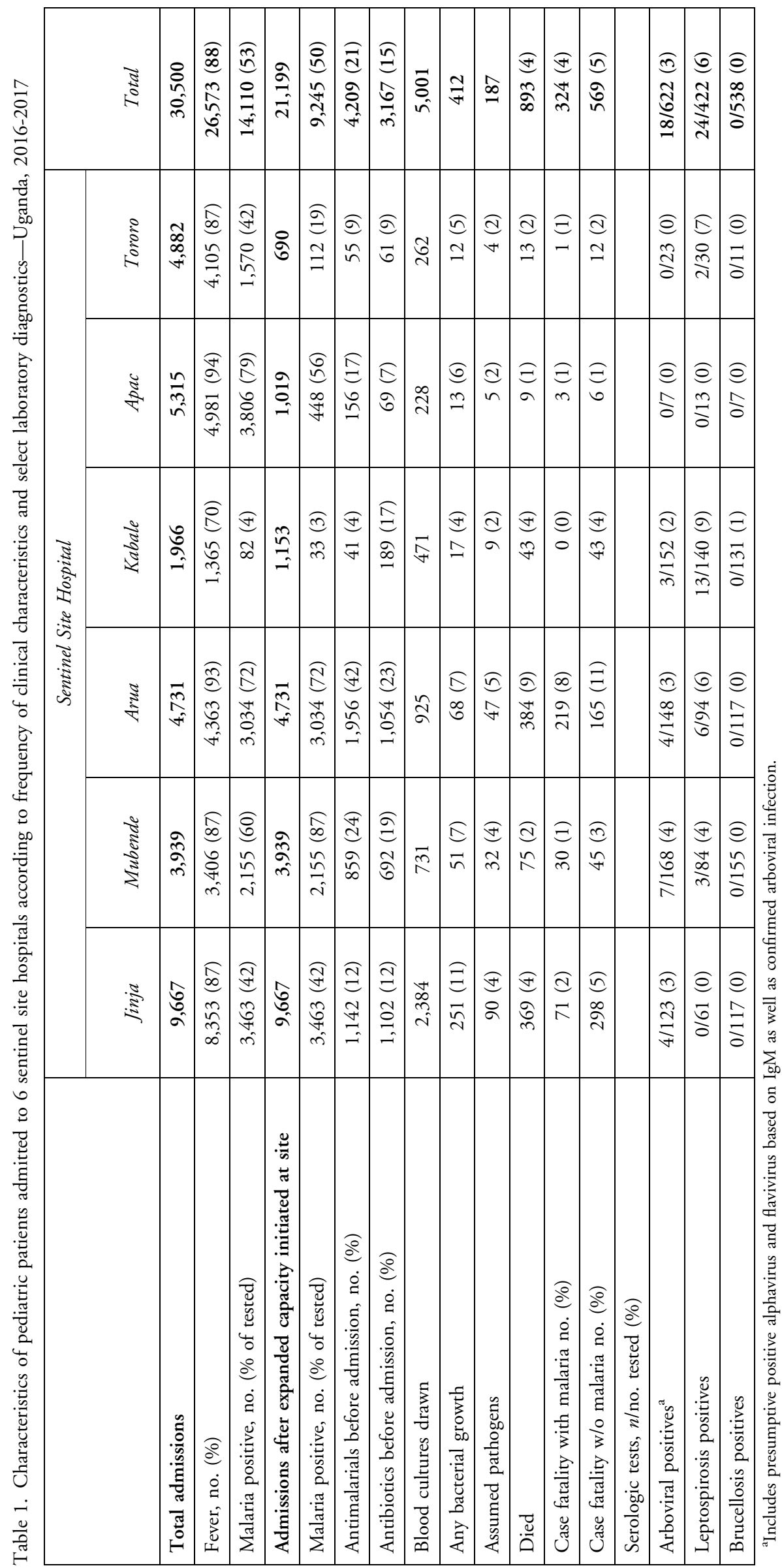




\section{CROSS-CUTTING APPROACH TO SURVEILLANCE TO IMPROVE HEALTH SECURITY IN UGANDA}

Table 2. Pathogenic organisms isolated from blood culture at 6 sentinel site hospitals_-Uganda, July 2016-December 2017

\begin{tabular}{|c|c|c|c|c|c|c|c|}
\hline \multirow[b]{2}{*}{$\begin{array}{l}\text { Microorganisms, no. } \\
\text { (column \%) }\end{array}$} & \multicolumn{7}{|c|}{ Number of Bacterial Isolates from Each Hospital, No. (\%) } \\
\hline & Jinja & Mubende & Arua & Kabale & Apac & Tororo & Total \\
\hline \multicolumn{8}{|l|}{ Gram negative } \\
\hline Acinetobacter spp. & $3(4)$ & $0(0)$ & $0(0)$ & $0(0)$ & $0(0)$ & $0(0)$ & 3 \\
\hline Citrobacter spp. & $7(8)$ & $2(8)$ & $2(5)$ & $1(17)$ & $0(0)$ & $0(0)$ & 12 \\
\hline Enterobacter spp. & $4(5)$ & $1(4)$ & $1(2)$ & $0(0)$ & $0(0)$ & $0(0)$ & 6 \\
\hline Escherichia coli & $5(6)$ & $1(4)$ & $2(5)$ & $1(17)$ & $0(0)$ & $0(0)$ & 9 \\
\hline Haemophilus spp. & $0(0)$ & $3(12)$ & $0(0)$ & $0(0)$ & $0(0)$ & $0(0)$ & 3 \\
\hline Klebsiella oxytoca & $1(1)$ & $0(0)$ & $0(0)$ & $0(0)$ & $0(0)$ & $0(0)$ & 1 \\
\hline Klebsiella pneumoniae & $8(10)$ & $0(0)$ & $0(0)$ & $0(0)$ & $0(0)$ & $0(0)$ & 8 \\
\hline Neisseria meningitidis & $0(0)$ & $1(5)$ & $0(0)$ & $0(0)$ & $0(0)$ & $0(0)$ & 1 \\
\hline Providencia spp. & $1(1)$ & $0(0)$ & $0(0)$ & $0(0)$ & $0(0)$ & $0(0)$ & 1 \\
\hline Pseudomonas aeruginosa & $3(4)$ & $0(0)$ & $0(0)$ & $0(0)$ & $0(0)$ & $0(0)$ & 3 \\
\hline Salmonella spp. & $7(8)$ & $10(45)$ & $34(81)$ & $1(17)$ & $3(100)$ & $0(0)$ & 55 \\
\hline Gram negative unidentified & & & & & & & 3 \\
\hline \multicolumn{8}{|l|}{ Gram-positive } \\
\hline Staphylococcus aureus & $17(20)$ & $2(9)$ & $0(0)$ & $1(17)$ & $0(0)$ & $3(75)$ & 23 \\
\hline Enterococcus spp. & $7(8)$ & $1(5)$ & $0(0)$ & $0(0)$ & $0(0)$ & $0(0)$ & 8 \\
\hline Streptococcus pneumoniae & $3(4)$ & $0(0)$ & $2(5)$ & $0(0)$ & $0(0)$ & $1(25)$ & 6 \\
\hline Streptococcus viridans ${ }^{\mathrm{a}}$ & $5(6)$ & $1(4)$ & $0(0)$ & $0(0)$ & $0(0)$ & $0(0)$ & 6 \\
\hline Gram positive unidentified & & & & & & & 12 \\
\hline TOTAL & 83 & 22 & 42 & 6 & 3 & 4 & 160 \\
\hline
\end{tabular}

${ }^{\mathrm{a} C}$ Considered a pathogen rather than contaminant here.

sites activated later in July and October 2017 (Table 1). Among these 21,199 admissions, 20\% $(n=4,209)$ self-reported antimalarial use and $15 \%(n=3,167)$ antibiotic use in the week before admission.

\section{Laboratory Data and Mortality}

A total of 5,001 single bottle blood cultures were performed at sentinel site hospitals through December 2017. Among these, 412 (8\%) yielded bacterial growth; 187 were deemed pathogenic bacteria by the processing laboratory. Of these 187 cultures, 160 had species identities available in the webbased surveillance system at the time of writing (Table 2). An additional 225 bottles grew likely contaminants, specifically Bacillus spp., coagulase-negative staphylococci, Rhodococcus spp., and Micrococcus spp.

A broad range of pathogens was isolated. The most commonly isolated pathogens were Salmonella spp. (34\%), Staphylococcus aureus (14\%), Citrobacter spp. (8\%), Escherichia coli (6\%), Enterococcus spp. (5\%), and Klebsiella pneumoniae (5\%) (Table 2). Notably, although the emphasis of the system was to improve diagnosis for non- malarial patients, and thus relatively few patients with malaria had blood cultures taken, pathogens were more commonly isolated from blood of malaria-positive children $(35 / 578 ; 6 \%)$ than from malaria-negative children (142/ 4258; 3\%) $(p=0.001)$.

Jinja RRH, east of Kampala, is the largest sentinel site in terms of patient volume and yielded the greatest diversity of pathogens (Table 2). Several of the pathogens identified to date originated commonly or exclusively from Jinja RRH, including E. coli, S. aureus, Pseudomonas aeruginosa, Enterococcus spp., Citrobacter spp., Acinetobacter spp., and Klebsiella pneumoniae. In contrast, among the 42 positive cultures isolated to date from Arua RRH, in the far northwest of Uganda, more than 70\% yielded Salmonella spp. (Table 2).

Following initiation of hospital-based leptospirosis testing, $6 \%$ of patient samples screened showed IgM reactivity on rapid test, with some potential for geographic clustering (Table 1). Arboviral screening of 622 sera yielded 18 (3\%) confirmed and presumptive-positive samples (Table 1). Fourteen samples were presumptive positive for recent flavivirus infection (eg, dengue, West Nile, yellow fever, or Zika). However, remaining sample volume was insufficient 
for PRNT confirmation of infecting virus. Positive samples originated from Jinja, Mubende, Arua, and Kabale. Of 4 samples with evidence of acute alphavirus infection, 2 were confirmed as o'nyong nyong virus infection, both originating from Arua. None of the sera screened for antibodies to Brucella spp. were positive. Among 293 patient sera screened in a Rickettsia africae IFA, 168 (57\%) demonstrated some degree of positive reactivity, with $22 \%$ of samples having reactivity at titers at or above 1:256 (data not shown).

Based on data from the processing laboratory, resistance to commonly used antimicrobials was observed in several bacterial species; a subset of these findings is presented in Table 3. Among S. aureus isolates tested, $71 \%$ were susceptible to clindamycin, $40 \%$ susceptible to erythromycin, $12 \%$ susceptible to penicillin; 0 of 10 isolates screened were susceptible to cotrimoxazole (Table 3). All $15 \mathrm{~S}$. aureus isolates screened against vancomycin were susceptible, but $25 \%$ screened were deemed methicillin resistant (MRSA) based on cefoxitin screen. S. pneumoniae isolates were susceptible to erythromycin, clindamycin, and chloramphenicol, but resistant to $\beta$ lactams (based on oxacillin screen). Salmonella spp. isolates demonstrated resistance to several agents, including ampicillin (39\% susceptible), cotrimoxazole (40\% susceptible), chloramphenicol (37\% susceptible), and ciprofloxacin (51\% susceptible) (Table 3); resistance to ceftriaxone was infrequently observed and only in nontyphoidal Salmonella. Other Enterobacteriaceae screened demonstrated resistance to thirdgeneration cephalosporins, as well as to imipenem (Table 3).

Overall, all-cause mortality among pediatric admissions at sentinel hospitals following availability of blood culture was $4 \%$, with a range across hospitals of $1 \%$ to $9 \%$ (Table 1 ). The case-fatality rate was $10 \%$ among those with positive blood cultures, 3\% among those that had negative blood cultures, and $4 \%$ among those with confirmed malaria.

\section{Discussion}

This sentinel surveillance and laboratory improvement effort in 6 Ugandan government hospitals demonstrates a pragmatic approach to identifying and tracking nonmalarial causes of febrile illness among hospitalized children aged 0 to 14 years. Early results from this effort led to the generation of more than 5,000 blood cultures, $4 \%$ of which yielded bacterial pathogens, including several WHO antimicrobial resistance priority pathogens, some with multidrug-resistant phenotypes, including Acinetobacter spp., Citrobacter spp., Escherichia coli, Staphylococcus aureus, and typhoidal and nontyphoidal Salmonella spp. Leptospirosis and arboviral infections (alphaviruses and flaviviruses) were also documented.

Although malaria is a common cause of fever in subSaharan Africa, including Uganda, there is increasing recognition that nonmalarial etiologies comprise a substantial portion of febrile illness. ${ }^{25,26}$ During the first several months of this effort in 6 hospitals, malaria accounted for approximately half of all pediatric febrile admissions. In- fectious etiologies among hospitalized children at these 6 government hospitals in Uganda are similar to that from other reports from the region, including varied bacterial blood stream pathogens, arboviruses, and leptospirosis. $^{3,5,27,28}$ Variability in the suite of pathogens across sites was evident, and further analyses should help identify baseline trends according to geography and season. Despite enhancement of laboratory capacity at these hospitals and centrally, the cause of illness for a large percentage of febrile admissions remain unknown. With over half of screened samples demonstrating reactivity to spotted fever group Rickettsia, it is clear that a large proportion of the pediatric population in Uganda is being exposed to these tickborne pathogens at an early age, and reliable diagnostics for acute rickettsial infections are needed in Uganda. Our efforts to improve routine diagnosis of infectious diseases began with basic blood culture and a few additional tests for select vector-borne and zoonotic conditions. With ongoing support, improved diagnostic capacity at the national level could include more complex techniques, or tests for additional pathogens that could be common causes of fever in East Africa. ${ }^{4}$ Ideally, this system will evolve into the foundation of an integrated approach to health care that enables clinicians to provide evidence-based care, including integration of HIV care and treatment. A local evidencebased approach to clinical care in Africa has the potential to be more cost-effective than an empirical, syndrome-based approach based on international guidelines. ${ }^{29}$

Challenges in interpretation of laboratory data in this setting remain evident. Antibiotic self-treatment was reported for $15 \%$ of pediatric admissions throughout Uganda during this 18 -month period, a frequency similar to that from other reports from Africa. ${ }^{27,30}$ Antibiotic pretreatment may have decreased sensitivity of blood cultures. Additionally, challenges obtaining adequate blood volumes, particularly from malnourished children, decreases the ability to subject clinical specimens to a wide array of diagnostic tests, potentially limiting diagnostic success. An investigation in early 2017 in Jinja RRH revealed that the majority of contaminants were from cultures drawn from children on the malnutrition ward. Some isolates deemed contaminants here could be causing illness, particularly in the malnourished or immunocompromised population. Moreover, some organisms isolated could represent hospital-acquired infections. However, the clinical and laboratory limitations inherent in this healthcare setting make it difficult to discern the clinical significance of these organisms. Despite these challenges, the overall proportion of pediatric blood cultures with evidence of bacterial pathogens was similar to that of other reports from Africa. 3,5,28,30,31 Antimicrobial susceptibility results presented here must also be interpreted with care. Reproducible AST results depend on the reagents used. The panel of antimicrobials tested varied with availability of reagents (or pharmaceuticals) and in some cases was expanded when resistance to first line drugs was observed. While these results may 


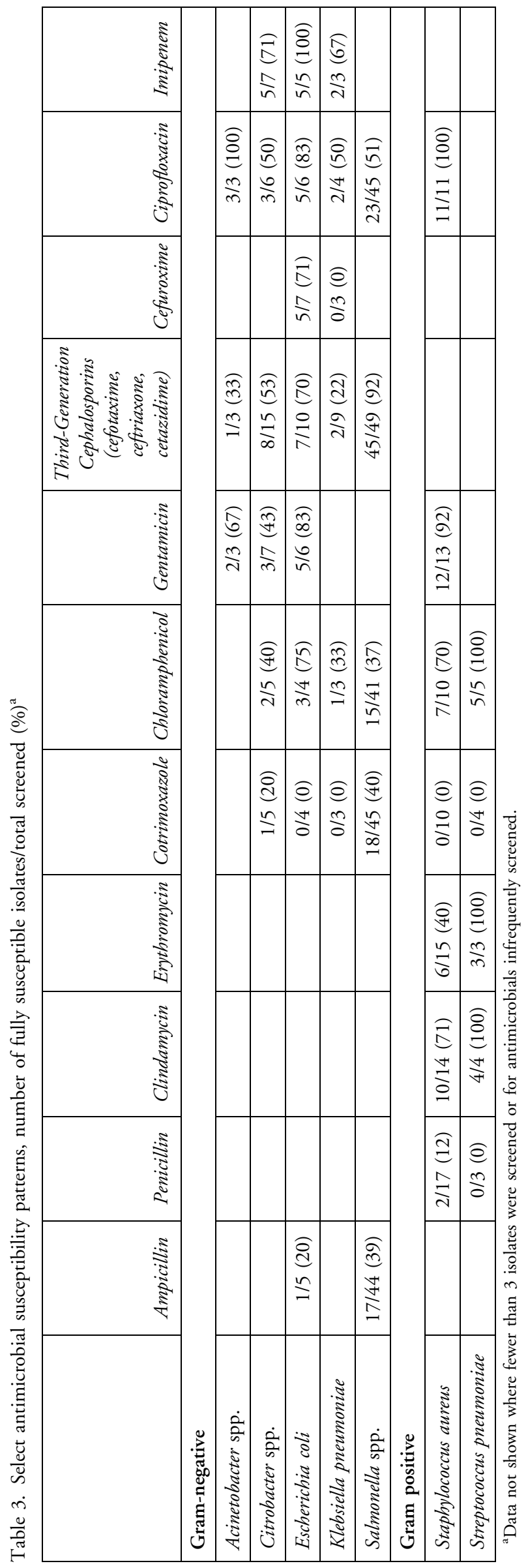


facilitate individual patient management and indicate overall trends, minimum inhibitory concentration testing against a panel of clinically and epidemiologically relevant drugs would be required for a comprehensive analysis.

Antibiotic misuse and lack of antimicrobial stewardship programs threaten the ability to treat historically treatable bacterial infections. ${ }^{32}$ Low- to middle-income countries may disproportionately feel the devastating consequences of drugresistant bacteria, as therapeutic options beyond traditional first line therapy may often be limited. Absent bacterial culture and susceptibility testing, empirical antimicrobial therapy may result in underuse or misuse of antibiotics in bacteremia or bacterial/malarial co-infections, and overuse in other undiagnosed viral febrile illnesses, thereby fomenting antimicrobial resistance. Gentamicin was the most commonly prescribed drug in these hospitals, and resistance to gentamicin was evident, particularly among E. coli, Klebsiella, and Citrobacter spp. Multidrug-resistant phenotypes and potential for nosocomial transmission highlight the urgent need for improved infection prevention and control in these healthcare settings. With increasing political recognition of the need to surveil for antimicrobial susceptibility, systems such as those described here will provide data needed to guide evidence-based intervention to mitigate spread of resistance and tailor treatment.

\section{Conclusion}

Data on routine and rare etiologies of febrile illness and associated trends improve not only health outcomes, but also the ability to detect and respond to unusual events of potential public health consequence. ${ }^{6}$ In Uganda, many disease-specific surveillance systems exist, such as for malaria, influenza, viral hemorrhagic fevers, and plague. ${ }^{15,33,34} \mathrm{~A}$ more comprehensive and integrated approach to disease surveillance and laboratory evaluation can generate information to minimize inefficiencies of disease-specific surveillance silos and assure that limited public health resources are leveraged appropriately to improve health security.

The system described here leverages prior surveillance investments to better identify common and rare causes of illness, generate antimicrobial susceptibility data, inform infection prevention and control interventions, and facilitate electronic surveillance infrastructure improvements. This approach to surveillance and laboratory systems improvement generates data and systems that can serve as the basis for responsive and sustainable public health surveillance in Uganda. Understanding of routine and rare causes of illness and development of appropriate laboratory capacity and associated systems is important to ensure systems in place are flexible and responsive to identify and detect epidemic-prone illness, whether existing or novel. In July 2017, a single case of Neisseria meningitidis infection was rapidly detected allowing for timely response that included tracking a patient who had left the hospital against medical advice, providing effective therapy, and providing prophylaxis to close contacts. The development of informatics systems infrastructure and associated workforce has served as a template for how case-based and event-based surveillance systems can be implemented and complement each other in Uganda. Implementation of nationwide electronic systems for Integrated Disease Surveillance and Response (eIDSR) is under way; associated processes were refined through the surveillance and laboratory capacity activities described here. Antimicrobial resistance data coming from these bacterial cultures are also informing Ugandan antimicrobial resistance task force actions. These cross-cutting improvements build knowledge and systems needed to enhance capacity to detect, report, and rapidly respond to public health concerns in Uganda, critical to compliance with International Health Regulations (IHR 2005).

\section{ACKNOWLedgments}

This work was supported through cooperative agreement number 5NU2GGH001744-02-00, funded by the US Centers for Disease Control and Prevention. Its contents are solely the responsibility of the authors and do not necessarily represent the official views of the Centers for Disease Control and Prevention or the Department of Health and Human Services.

\section{REFERENCES}

1. Prasad N, Sharples KJ, Murdoch DR, Crump JA. Community prevalence of fever and relationship with malaria among infants and children in low-resource areas. Am J Trop Med Hyg 2015;93(1):178-180.

2. Crump JA, Kirk MD. Estimating the burden of febrile illnesses. PLoS Negl Trop Dis 2015;9(12):e0004040.

3. Crump JA, Morrissey AB, Nicholson WL, et al. Etiology of severe non-malaria febrile illness in Northern Tanzania: a prospective cohort study. PLoS Negl Trop Dis 2013;7(7):e2324.

4. D’Acremont V, Kilowoko M, Kyungu E, et al. Beyond malaria-causes of fever in outpatient Tanzanian children. $N$ Engl J Med 2014;370(9):809-817.

5. Prasad N, Murdoch DR, Reyburn H, Crump JA. Etiology of severe febrile illness in low- and middle-income countries: a systematic review. PLoS One 2015;10(6):e0127962.

6. Petti CA, Polage CR, Quinn TC, Ronald AR, Sande MA. Laboratory medicine in Africa: a barrier to effective health care. Clin Infect Dis 2006;42(3):377-382.

7. World Bank. Uganda Country Profile 2018. http://www.world bank.org/en/country/uganda. Accessed September 7, 2018.

8. de St Maurice A, Nyakarahuka L, Purpura L, et al. Notes from the field: Rift Valley Fever response-Kabale District, Uganda, March 2016. MMWR Morb Mortal Wkly Rep 2016; 65(43):1200-1201.

9. Wamala JF, Lukwago L, Malimbo M, et al. Ebola hemorrhagic fever associated with novel virus strain, Uganda, 20072008. Emerg Infect Dis 2010;16(7):1087-1092. 
10. Wamala JF, Malimbo M, Okot CL, et al. Epidemiological and laboratory characterization of a yellow fever outbreak in northern Uganda, October 2010-January 2011. Int J Infect Dis 2012;16(7):e536-e542.

11. Kwesiga B, Pande G, Ario AR, Tumwesigye NM, Matovu JKB, Zhu BP. A prolonged, community-wide cholera outbreak associated with drinking water contaminated by sewage in Kasese District, western Uganda. BMC Public Health 2017;18(1):30.

12. Walters MS, Routh J, Mikoleit M, et al. Shifts in geographic distribution and antimicrobial resistance during a prolonged typhoid fever outbreak-Bundibugyo and Kasese Districts, Uganda, 2009-2011. PLoS Negl Trop Dis 2014;8(3):e2726.

13. Begier EM, Asiki G, Anywaine Z, et al. Pneumonic plague cluster, Uganda, 2004. Emerg Infect Dis 2006;12(3):460-467.

14. Knust B, Schafer IJ, Wamala J, et al. Multidistrict outbreak of Marburg Virus disease-Uganda, 2012. J Infect Dis 2015; 212(Suppl 2):S119-S128.

15. Shoemaker TR, Balinandi S, Tumusiime A, et al. Impact of enhanced viral haemorrhagic fever surveillance on outbreak detection and response in Uganda. Lancet Infect Dis 2018; 18(4):373-375.

16. Teshale EH, Howard CM, Grytdal SP, et al. Hepatitis E epidemic, Uganda. Emerg Infect Dis 2010;16(1):126-129.

17. Wamala JF, Okot C, Makumbi I, et al. Assessment of core capacities for the International Health Regulations (IHR[2005])—Uganda, 2009. BMC Public Health 2010; 10(Suppl 1):S9.

18. Braden CR, Dowell SF, Jernigan DB, Hughes JM. Progress in global surveillance and response capacity 10 years after severe acute respiratory syndrome. Emerg Infect Dis 2013; 19(6):864-869.

19. Borchert JN, Tappero JW, Downing R, et al. Rapidly building global health security capacity-Uganda demonstration project, 2013. MMWR Morb Mortal Wkly Rep 2014; 63(4):73-76.

20. Potter C, Brough R. Systemic capacity building: a hierarchy of needs. Health Policy Plan 2004;19(5):336-345.

21. World Health Organization. WHO Guide for the Stepwise Laboratory Improvement Process Towards Accreditation (SLIPTA) in the African Region. WHO AFRO; 2015. https://www.afro.who. int/publications/who-guide-stepwise-laboratory-improvementprocess-towards-accreditation-slipta-african. Accessed September 7, 2018.

22. World Health Organization. WHO Laboratory Assessment Tool. Geneva: WHO; 2012. http://www.who.int/ihr/publications/ laboratory_tool/en/. Accessed September 7, 2018.

23. Centers for Disease Control and Prevention. Infection control assessment tools. CDC website. Updated February 15, 2017. https://www.cdc.gov/hai/prevent/infection-controlassessment-tools.html. Accessed September 7, 2018.
24. Brown SL, Klein GC, McKinney FT, Jones WL. Safranin Ostained antigen microagglutination test for detection of Brucella antibodies. J Clin Microbiol 1981;13:398-400.

25. Sears D, Mpimbaza A, Kigozi R, et al. Quality of inpatient pediatric case management for four leading causes of child mortality at six government-run Ugandan hospitals. PLoS One 2015;10(5):e0127192.

26. Nankabirwa J, Zurovac D, Njogu JN, et al. Malaria misdiagnosis in Uganda-implications for policy change. Malar J 2009;8:66.

27. Reddy EA, Shaw AV, Crump JA. Community-acquired bloodstream infections in Africa: a systematic review and meta-analysis. Lancet Infect Dis 2010;10(6):417-432.

28. Marks F, von Kalckreuth V, Aaby P, et al. Incidence of invasive salmonella disease in sub-Saharan Africa: a multicentre population-based surveillance study. Lancet Glob Health 2017;5(3):e310-e323.

29. Penno EC, Baird SJ, Crump JA. Cost-effectiveness of surveillance for bloodstream infections for sepsis management in low-resource settings. Am J Trop Med Hyg 2015;93(4): 850-860.

30. Nichols C, Cruz Espinoza LM, von Kalckreuth V, et al. Bloodstream infections and frequency of pretreatment associated with age and hospitalization status in Sub-Saharan Africa. Clin Infect Dis 2015;61(Suppl 4):S372-S379.

31. Mahende C, Ngasala B, Lusingu J, et al. Aetiology of acute febrile episodes in children attending Korogwe District Hospital in north-eastern Tanzania. PLoS One 2014;9(8):e104197.

32. World Health Organization. Prioritization of Pathogens to Guide Discovery, Research and Development of New Antibiotics for Drug-Resistant Bacterial Infections, Including Tuberculosis. Geneva: WHO; 2017. http://www.who.int/medicines/areas/ rational_use/prioritization-of-pathogens/en/. Accessed September 7, 2018.

33. Forrester JD, Apangu T, Griffith K, et al. Patterns of human plague in Uganda, 2008-2016. Emerg Infect Dis 2017;23(9): 1517-1521.

34. Cummings MJ, Bakamutumaho B, Kayiwa J, et al. Epidemiologic and spatiotemporal characterization of influenza and severe acute respiratory infection in Uganda, 2010-2015. Ann Am Thorac Soc 2016;13(12):2159-2168.

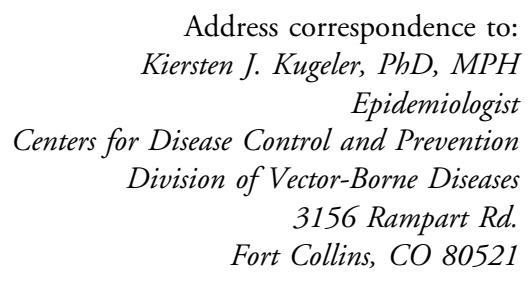

Email: bio1@cdc.gov 